IC Vol. $5 N^{\circ}$ 2: pp. 233-251, 2014

\title{
LOS RECURSOS TIC FAVORECEDORES DE ESTRATEGIAS DE APRENDIZAJE AUTÓNOMO: EL ESTUDIANTE AUTÓNOMO Y AUTORREGULADO*
}

\author{
THE TIC RESOURCES FAVORING INDEPENDENT LEARNING \\ STRATEGIES: STUDENT SELF-REGULATED AND SELF-
}

\author{
Juan Roger Rodríguez Ruiz ${ }^{1}$
}

\section{RESUMEN}

El desarrollo de la presente investigación sobre el estudiante autónomo y autorregulado permitió verificar el impacto que las nuevas Tecnologías de la Información y Comunicación han generado nuevas necesidades laborales y, por ende, educativas. Nuestro objetivo fue verificar cómo los recursos TIC favorecen las estrategias del aprendizaje autónomo y autorregulado del estudiante. Nuestra investigación buscó responder a la necesidad de aumentar la autonomía del estudiante en su propio proceso de aprendizaje a través de diferentes recursos TIC, de tal manera que esté en capacidad de relacionar problemas por resolver y destrezas por desarrollar con necesidades y propósitos de aprendizaje, así como de buscar la información necesaria, analizarla, generar ideas para solucionar problemas, sacar conclusiones y establecer el nivel de logro de sus objetivos. Las estrategias de aprendizaje autorreguladas como acciones y procesos dirigidos influyeron en los estudiantes autónomos y autorregulados, que se caracterizan por tener diversas capacidades cognitivas y metacognitivas. La capacidad de autorregulación implicó la ayuda social y el uso activo de diferentes recursos que posibilitaron el logro académico esperado y el propio proceso de aprendizaje. Los recursos TIC considerados en esta investigación (la pizarra digital, los WebQuest, las actividades interactivas y tutoriales electrónicas y los trabajos colaborativos: La Web 2.0, ofrecieron ventajas para el aprendizaje autorregulado.

PALABRAS CLAVE: Estrategias de aprendizaje, estudiante autónomo y autorregulado, recurSOs TIC.

* Recibido: 19 agosto 2014; aprobado: 21 noviembre 2014.

1 Doctor en Derecho Canónico por la Pontificia Universidad Gregoriana de Roma. Jefe de Publicaciones de la Universidad Católica Los Ángeles de Chimbote, Perú. qahais@yahoo.com.ar 


\section{ABSTRACT}

The development of this research on the autonomous and self-regulated student allowed to verify the impact of new information and communication technologies have created new work requirements and therefore educational. It aimed to verify how ICT resources promote independent learning strategies and self-regulated in the student. This research sought to address the need to increase the autonomy of students in their own learning process through different ICT resources, so that it is able to relate problems to solve and develop skills with needs and purposes of learning, and find the necessary information, analyze it, generate ideas to solve problems, draw conclusions and establish the level of achievement of its objectives. Self-regulated learning strategies as actions and directed processes influenced autonomous and self-regulated students that are typical for their various cognitive and metacognitive skills. The self regulation involved social support and the active use of different resources that enabled the expected academic achievement and the learning process. The ICT resources considered in this research were: the whiteboard, the WebQuest, the interactive activities, the electronic tutorials and the collaborative work; The Web 2.0 offered advantages for self-regulated learning.

KEY WORDS: Learning strategies, autonomous and self-regulated student, ICT resources.

\section{INTRODUCCIÓN}

El tema del estudiante autónomo y autorregulado nos permite ver el impacto que las nuevas tecnologías de la información y comunicación han generado en el mundo entero nuevas necesidades laborales y por ende educativas.

Cada vez más hay una tendencia fuerte y radical de desplazamiento de las prácticas pedagógicas hacia un aprendizaje centrado en el estudiante y, por tanto, se presenta un cambio en el papel del maestro: ya no se dedica a transmitir conocimientos o a instruir, sino a orientar el proceso educativo en ambientes en los cuales el estudiante sea capaz de identificar y decidir lo que quiere aprender y las condiciones en que va a hacerlo.

En otras palabras, se ha venido dando mayor importancia al aprendizaje autodirigido, en el cual el estudiante debe ser capaz, tanto de identificar sus necesidades de aprendizaje como de acudir a las fuentes de información y a procesos de formación para satisfacer dichas necesidades. Bajo esta concepción el aprendizaje no se limita a las cuatro paredes del aula, ya que los límites se amplían a todos los escenarios en que interactúa el estudiante; es decir, se tiene el mundo entero por aula de clases. Este tipo de aprendizaje se viene aplicando ampliamente en procesos de instrucción relacionados con la formación profesional en diversas disciplinas, el desarrollo de recursos humanos en la empresa, la educación continuada, etc. Este cambio explica, en parte, la aceptación gene- 
ralizada de principios básicos como los siguientes: el reconocimiento de que cada persona aprende conceptos y desarrolla destrezas de manera distinta y a ritmo diferente que otros estudiantes; el aprendizaje es más efectivo cuando es experimental, o sea que aprendemos mejor cuando tenemos la oportunidad de probar o de confrontar con la realidad los conceptos teóricos. Como respuesta a los incesantes cambios que se presentan en todos los ámbitos, el aprendizaje debe ser permanente, o sea que se realiza durante toda la vida; por tanto, toda persona debe desarrollar habilidades para la adquisición de nuevos conocimientos de manera continua e independiente.

La siguiente propuesta busca responder a esta necesidad de aumentar la autonomía del estudiante en su propio proceso de aprendizaje a través de diferentes recursos TIC, de tal manera que esté en capacidad de relacionar problemas por resolver y destrezas por desarrollar con necesidades y propósitos de aprendizaje, así como de buscar la información necesaria, analizarla, generar ideas para solucionar problemas, sacar conclusiones y establecer el nivel de logro de sus objetivos.

\section{DESARROLLO TEMÁTICO}

\section{AUTONOMÍA Y AUTORREGULACIÓN}

La autorregulación puede definirse como la capacidad que una persona adquiere para orientar su propia conducta (López, 2008).

Se debe tener en consideración que existen múltiples maneras de concebir y entender la autonomía en el aprendizaje y la dificultad de llegar a una concepción común. La reflexión sobre nuestra práctica desveló el dilema entre la libertad total ("laissez faire") y el control estricto que experimenta el profesorado al proponer contextos de aprendizaje autónomo. Este dilema se plantea al momento de decidir entre dar al alumnado completa libertad para elegir los objetivos y estrategias de aprendizaje a seguir, o bien dejar esta decisión exclusivamente en manos del profesorado.

También debemos tener presente el concepto de autodirección como capacidad que tiene el estudiante adulto de asumir la responsabilidad de la planificación y dirección del curso de aprendizaje. Las personas que toman la iniciativa en el aprendizaje tienen más responsabilidad de retener lo aprendido. En la autonomía hay más facilidad para mantenerse motivado en el proceso de aprendizaje. La piedra angular para el aprendizaje autodirigido es la responsabilidad personal. 


\subsection{APRENDIZAJE AUTÓNOMO Y AUTORREGULADO}

En el contexto del aprendizaje, la autorregulación consiste básicamente en formularse metas concretas, planificar actividades para el logro de esas metas, monitorear el desempeño durante la ejecución de tales actividades, evaluarse continuamente de acuerdo con las metas y criterios fijados y, finalmente, valorar el producto del proceso de aprendizaje.

El concepto de autorregulación fue introducido en 1971 por Bandura en su “Teoría del aprendizaje social” (Bandura, 1971), quien la consideró como resultado del proceso de socialización en cada individuo. El sujeto aprende a ejecutar una serie de conductas que observa en otros por medio un proceso que se llama modelado. Al principio, estas conductas aprendidas se refuerzan mediante consecuencias externas (por condicionamiento operante). Más adelante, este refuerzo deja de ser necesario y el sujeto es capaz de ejecutar esas conductas sin que exista un modelo o un refuerzo; es decir, el sujeto es regulado externamente en una primera fase y, poco a poco, internaliza está regulación para, finalmente, ser capaz de regularse por sí mismo.

En el entorno escolar, los estudiantes que se autorregulan son promotores activos de su propio proceso de aprendizaje y de su rendimiento académico. Esto se logra a través de la puesta en práctica de una serie de estrategias cognitivas, metacognitivas, motivacionales y conductuales (Zimmerman, 1986).

La utilización deliberada de estas estrategias no solo permite al sujeto construir conocimiento de forma significativa, sino que conlleva un autoconocimiento respecto de las formas individuales más eficaces de utilizar o aplicar lo aprendido.

Sobre el planteamiento anterior, el estudiante podría autorregular tres dimensiones en el aprendizaje: la cognición, la motivación y la conducta observable.

Respecto de la dimensión cognitiva, el sujeto podría conocer y manejar una serie de estrategias cognitivas y metacognitivas para llevar a cabo las tareas e, igualmente, podría conocerse a sí mismo como procesador de la información y saber qué necesita, en términos de memoria, atención o conocimiento previo, para aprender de manera eficaz.

En lo referente a la motivación, los estudiantes que lograsen autorregular su aprendizaje serían capaces de controlar y hacer más realistas sus creencias personales sobre lo que son capaces de hacer cuando se enfrentan a una tarea $o$ sobre el tipo de metas que pueden formularse frente a una situación de aprendizaje. 
Por último, si se logra la autorregulación de la conducta, ello implicaría convertirse en una persona activa para crear ambientes que optimicen el propio aprendizaje, para encontrar lugares adecuados de estudio y para buscar ayuda de terceros cuando se requiera (Pintrich, 1995).

Si se quiere hablar de autorregulación en el aprendizaje, es necesario que exista la posibilidad de elección y control por parte del estudiante; es decir, es importante que sea el propio estudiante quien ejerza control, haga elecciones y autoinicie conductas y no sólo actué en respuesta a los requerimientos de otros.

Conforme a este criterio, la eficacia del entrenamiento en estrategias de autorregulación sólo se podría determinar cuando los estudiantes tengan la posibilidad de aprender de la forma como ellos elijan. Bajo esta lógica, es de especial interés determinar hasta qué punto logra un estudiante darle una valoración personal a lo que está aprendiendo y, en consecuencia, es capaz de autoiniciar y autocontrolar un proceso de aprendizaje (Zimmerman, 1994).

Así la situación, se parte del supuesto de que la autorregulación puede enseñarse y que no se adquiere de una vez y para siempre, sino que pasa por distintas etapas superables mediante la orientación y la práctica repetidas, a través de múltiples experiencias en diferentes contextos.

La mayoría de las definiciones de aprendizaje autorregulado destacan la importancia de la autonomía, el autocontrol y la autodirección, refriéndose al sujeto como promotor activo o autogenerador de procesos y conductas en las tres dimensiones mencionadas (cognitiva, motivacional y conductual).

Además, coinciden en identificar que los sujetos que autorregulan su aprendizaje tienen mayores logros académicos que los que no se autorregulan (Pintrich, 1986).

\subsection{ESTUDIANTE AUTÓNOMO Y AUTORREGULADO}

En general, se puede describir a los estudiantes como autorregulados en la medida en que ellos sean participantes metacognitiva, motivacional y conductualmente activos en sus propios procesos de aprendizaje (Zimmerman, 1986).

Tales estudiantes personalmente inician y dirigen sus propios esfuerzos para adquirir conocimientos y habilidades en vez de confiar en profesores, padres y otros agentes de instrucción.

Para ser considerados como autorregulados, el aprendizaje de los estudiantes debe involucrar el uso de estrategias específicas para conseguir objetivos académicos basadas en percepciones de auto-eficacia

Esta definición asume la importancia de tres elementos: 
a) Estrategias de aprendizaje autorreguladas de los estudiantes;

b) Percepciones de auto-eficacia sobre las habilidades de ejecución; y

c) Compromiso con los logros académicos.

Las estrategias de aprendizaje autorreguladas son acciones y procesos dirigidos hacia la adquisición de información o habilidades que involucran gestión, propósito y percepciones instrumentalizadas por los estudiantes.

Estas incluyen métodos organización y transformación de la información, búsqueda autoconsecuente de información, reescucha o el uso de ayuda nemotécnica.

La autoeficacia (Bandura, 1982) se refiere a percepciones acerca de las propias capacidades para organizarse e implementar las acciones necesarias para obtener las habilidades de ejecución designadas para tareas específicas.

Objetivos académicos, tales como notas, estima social u oportunidades de empleo después de la graduación pueden variar ampliamente en naturaleza y en tiempo de logro; lo importante es la valoración que le da el estudiante a estos: si es una valoración alta, entonces el compromiso en la adquisición de estos será obviamente mayor.

Esta definición se centra en cómo los estudiantes representan acciones y condiciones actuales en función de estrategias para la obtención de objetivos subsecuentes, y asume una orientación motivacional; de parte de los estudiantes es mantenida por continuas auto-percepciones de eficacia en la realización de una tarea específica.

Por lo tanto, para que se puedan describir como autorreguladas las acciones estratégicas de los estudiantes se debe conocer primero sus objetivos académicos y sus percepciones de eficacia.

Por ejemplo, uno no puede definir el hecho de que un estudiante salga a tomar café después de estudiar como una estrategia autoconsecuente sin saber su propósito para usar esta estrategia (por ejemplo, para mejorar la motivación) y sus percepciones de eficacia cuando lo usa (por ejemplo, hacer más trabajo).

\subsection{TEORÍA DEL APRENDIZAJE AUTORREGULADO}

Diversas investigaciones (Alexander, 2004) en el campo de psicología educacional han examinado qué procesos poseen los estudiantes exitosos, procesos específicamente relacionados con actividades estratégicas, monitoreadas y planeadas.

Durante la década de 1970 las investigaciones revelaron que estudiantes exitosos usaron procesos de observancia y planeamiento que eran fundamental- 
mente diferentes de la de sus pares que fueron menos exitosos académicamente en la escuela. Estos procesos estuvieron caracterizados por tener componentes autorregulatorios (París, 1990).

Las más actuales investigaciones en aprendizaje autorregulado (Moos, 2007) se han concentrado en aprendizaje aislado, mientras que las aproximaciones al aprendizaje autorregulado en la década de 1980 presentan modelos más comprensivos y multifacéticos. Estos modelos de aprendizaje autorregulado ofrecen una perspectiva que consideró a estudiantes que aprenden preactiva y estratégicamente como opuestos a estudiantes en sus ambientes.

Para explicar esta orientación preactiva y estratégica, los investigadores apelaron a variables sociales, conductuales, motivacionales y cognitivas en diferentes contextos de instrucción. Estos modelos de aprendizaje autorregulado han evolucionado en los últimos 20 años, debido, en parte, a la considerable investigación examinando el aprendizaje autorregulado en los logros académicos.

Recientemente, los investigadores han usado la teoría de autorregulación del aprendizaje para examinar cómo los estudiantes aprenden en ambientes de aprendizaje basados en computadoras, tales como ambientes hipermedia (Moos, 2007).

\subsection{ESTUDIANTE AUTÓNOMO}

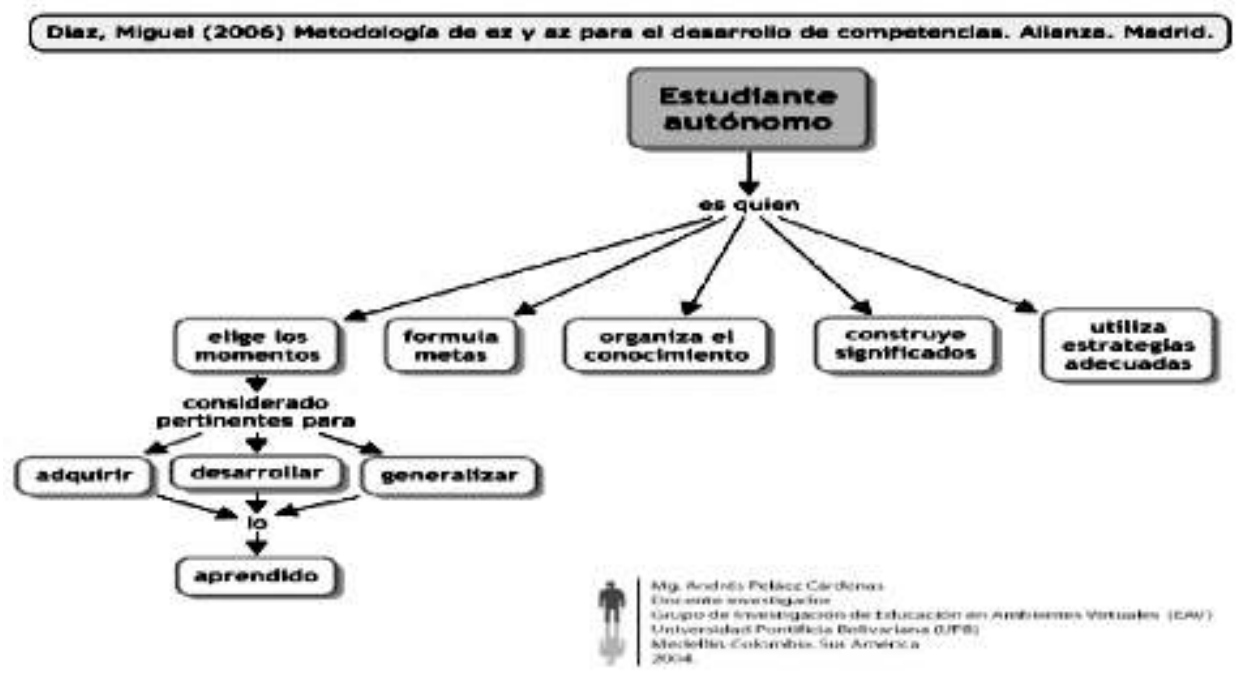

Gráfico 1. Estudiante autónomo. 
En el diccionario de la Academia de la Lengua Española aparece que la acepción o uso del término "autonomía" aplicada a personas refiere la condición de quien, para ciertas cosas, no depende de nadie. Y "depender" es entendido como producirse o ser causado por alguien o algo. Integrando ambos significados, aprendizaje autónomo o autoaprendizaje sería el conseguido por el individuo sin el concurso de otros, sin estar causado por un agente personal o material distinto al sujeto del aprendizaje.

Brockett y Hiemtra desarrollan el concepto de aprendizaje autodirigido en lugar de aprendizaje autónomo. Al respecto, afirman que "la autodirección en el aprendizaje es una combinación de fuerzas tanto interiores como exteriores de la persona que subrayan la aceptación por parte del estudiante de una responsabilidad cada vez mayor respecto a las decisiones asociadas al proceso de aprendizaje" (Brocket, 1993).

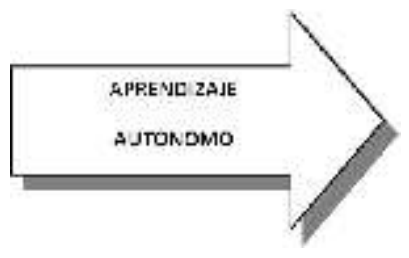

Implica mayor intervención del estudiante en la determinación de:

口 Objetivos.

口 Procedimientos.

Recursos.

Evaluaciones.

- Momentos de aprendizaje.

Cuando se habla de aprendizaje autónomo, se está haciendo referencia al grado de participación e intervención del estudiante en el establecimiento y desarrollo de sus propios objetivos, procedimientos, recursos, evaluación y momentos de aprendizaje. Lo anterior indica que cuando el estudiante participa en forma más directa en las decisiones que afectan su propio aprendizaje, la motivación y efectividad en su proceso de aprendizaje se hacen más fáciles y asimilables.

Una forma para entender el aprendizaje autónomo es comprenderlo como “...el proceso mediante el cual una persona adquiere destrezas o habilidades prácticas (motoras o intelectuales), incorpora contenidos informativos o adopta nuevas estrategias de conocimientos y/o acción" (Garza, 1998).

Para lograr las competencias de ser un profesional de alta calidad y competitividad, el estudiante debe amar su estudio, entregarse y acoger las estrategias más apropiadas para llegar a donde desea llegar. Otro concepto que debe ser comprendido es cómo se interpreta la autonomía. Aquí significa "la competen- 
cia de cada cual de comportarse de acuerdo con su concepción de la vida, del bien, de la sociedad y de la historia" (Hoyos, 1998).

La autonomía es la capacidad que tiene cada persona para orientar y justificar sus acciones; por lo tanto, "ilumina... la situación y al mismo tiempo capacita para poder obrar adecuadamente siendo conscientes de nuestras propias competencias".

Teniendo en cuenta los planteamientos anteriores, el aprendizaje autónomo se podría definir, como la capacidad de las personas para aprender a aprender, aprender a compartir, aprender a evaluar y valorar, aprender a cambiar y aprender a mejorar; en otras palabras, cumplir con los pilares de la educación propuesta por la comisión internacional sobre la educación para el siglo XXI de la UNESCO (Malagón, 2005).

Desde esta perspectiva, el desarrollo de competencias para el aprendizaje requiere del aprendiente o estudiante de aprender a aprender, que según Insuasty "significa que la persona ha desarrollado habilidades y estrategias cognitivas que le permiten procesar información eficientemente y de acuerdo con las necesidades del momento" (Insuasty, 1997).

Las actividades de aprendizaje autónomo promueven la capacidad de aprender de manera continua fuera de un contexto escolar tradicional. Mediante un diseño apropiado de las actividades, se propicia en los estudiantes la reflexión respecto de sus propios procesos de aprendizaje, lo que contribuye a desarrollar la capacidad de aprender por cuenta propia mediante la construcción de:

- Hábitos de estudio, tales como: concentración, disciplina, búsqueda de información.

- Habilidades de comprensión de lectura.

- La habilidad de buscar información necesaria cuando sea un tema que se desconozca o se desee profundizar en él.

- La selección de la información importante y significativa para los propósitos que se propone alcanzar.

- El análisis de la información con espíritu interpretativo, propositivo, crítico, analítico y argumentativo.

\section{CARACTERÍSTICAS DEL APRENDIZAJE AUTÓNOMO}

Se retoma la definición del aprendizaje autónomo o autoaprendizaje como el que sería conseguido por el individuo sin el concurso de otros, sin estar causado por un agente personal o material distinto al sujeto del aprendizaje (Moreno y Rafael, 2007). 
Dicho significado inicial permite derivar algunos aspectos y características:

Primero, la independencia definitoria de la autonomía de un determinado aprendizaje se entiende con respecto a determinados referentes; "para ciertas cosas", según la definición citada, tales como espacio, tiempo, personas y materiales elaborados por estas, distintas al propio sujeto del aprendizaje.

Segundo, la independencia puede ser considerada en grados o niveles más que como una cuestión de todo o nada. Un aprendizaje puede resultar, por tanto, más o menos autónomo o independiente de un determinado referente.

Tercero, al poder considerar distintos referentes y dominios de los que se independiza el aprendizaje, el grado de autonomía de este puede ser distinto respecto a cada uno de ellos; un aprendiz puede ser más autónomo en matemáticas que en lenguaje, y también, en algunos temas de cada materia más que en otros.

Cuarto, expresar la autonomía en términos de independencia es hacerla en términos negativos, especificando los referentes que han dejado de ser relevantes; por ello conviene completar la noción en términos positivos de la dependencia que también implica, evitando el sinsentido de un aprendizaje "a-causado", sin dependencia alguna. La autonomía con respecto a referentes ajenos propia del autoaprendizaje ha de verse sustituida así por la dependencia, fundamentalmente con respecto a las propias acciones del aprendiz. Y decimos fundamental y no exclusivamente, porque el máximo de autonomía existente cuando el sujeto del aprendizaje prescindiera de todo elemento no generado por él y funcionara con completa independencia del medio, solo se daría -si es que ocurre- en personas altamente cualificadas en algún dominio determinado.

Quinto, el logro de la autonomía en el aprendizaje puede entenderse como un proceso doble, complementario y progresivo: aumento de la independencia con respecto a referentes ajenos al individuo y aumento de la dependencia de las actividades del propio individuo hasta llegar a prescindir de lo ajeno o al menos a interpretarlo de manera personal.

Sexto, descrito con diferentes matices y énfasis por autores como Piaget,1975, Ribes y López, 1985, Vygotsky, 1979, tal proceso comienza con la independencia respecto a las relaciones constantes y limitadas en número marcadas por el equipamiento biológico con el que nace el individuo, pasando en su experiencia a relacionarse progresivamente con un mayor número y variedad de situaciones, lo que implica ir pasando a depender en menor medida de cada una de ellas en particular. Por ejemplo, cuando se ha aprendido a operar algebraica- 
mente en forma adecuada, se depende menos de cómo es cada nueva operación a resolver -como por ejemplo en el número y complejidad de sus elementos, o con qué anotación y en qué contexto están presentados- y también de una determinada manera fija de resolverlas -mentalmente, con calculadora o algún otro recurso-, adaptándose por tanto a la variedad de situaciones que se puedan encontrar.

En ese proceso, alcanzar aprendizajes más autónomos requiere, por tanto, partir de los más heterónomos logrados en la historia individual de cada sujeto.

Séptimo, la culminación de la autonomía ocurriría con los niveles superiores de ese desarrollo e implicaría la integración de repertorios previos ahora relacionados, siendo capaz primero el individuo de describir a otros y a sí mismo el propio comportamiento, y posteriormente, de abstraer reglas resúmenes sobre sus propias experiencias, lo que le permite su aplicación a nuevas situaciones y circunstancias consideradas entonces como casos particulares de las reglas obtenidas. Ambos desempeños son necesariamente lingüísticos y convencionales, lo que significa que el aprendizaje autónomo de mayor nivel es algo propio de los humanos.

Octavo, asumiendo la definición de autonomía en términos funcionales de independencia-dependencia, no resulta lo más útil ni significativo identificarla en términos de indicadores morfológicos, como escribir, leer textos, exponer un trabajo en público, trabajar en grupo o cualquier otro en términos de apariencia de las conductas del organismo u objetos del entorno implicados. Y es que una misma morfología puede implicar grados diversos de autonomía funcional.

Exponer un trabajo en público puede suponer la tarea poco autónoma de leer notas recibidas de otras personas o las más autónomas realizadas sin dependencia de notas de otros o escritas previamente por uno mismo incluso. Igual ocurre con la resolución de ejercicios identificada morfológicamente por realizarse en ausencia del profesor; podría implicar la tarea de bajo nivel de autonomía de reconocer mediante claves visuales, como color o forma, imágenes histológicas aprendidas previamente; pero también, un mayor nivel de autonomía si la resolución de los ejercicios exige evaluaciones con diversos criterios a relacionar entre sí. En todo caso, los criterios morfológicos pueden ser útiles como indicadores iniciales de aprendizajes cuyo nivel de autonomía debe ser identificado; pero no deberían ocultar o sustituir a los indicadores del grado de independencia y dependencia respecto a determinados referentes.

Noveno, todo aprendizaje incluido el autónomo, se refiere a un determina- 
do contenido; es decir, es válido para determinados dominios o ámbitos de práctica social. Por ello son posibles aprendizajes autónomos en los que el logro sea el propio aprendizaje de cómo lograr otros aprendizajes. En ellos, el individuo es capaz de organizarse a sí mismo las circunstancias favorecedoras del cumplimiento de logro en algún contenido específico, bien describiéndose las interacciones con su medio que debe establecer en un determinado dominio, bien formulando además una regla válida para uno o más dominios. Ello es lo que supone aprender a aprender o enseñarse a sí mismo, o sea la autoenseñanza.

Décimo, aunque el aprender a aprender implica siempre los niveles altos de autonomía recién mencionados, el aprendizaje que el propio individuo se facilita puede ser de cualquiera de los niveles. Podrá aprender a lograr aprendizajes sencillos al establecer asociaciones necesarias para un determinado logro, como, por ejemplo, cuando se va diciendo a sí mismo los pasos de un proceso de habilidad mecánica. Pero también puede diseñarse el logro de aprendizajes más autónomos, como organizar reglas metodológicas que permitan el aprendizaje de tareas necesarias para realizar investigaciones válidas.

\subsection{CARACTERÍSTICAS DE LOS ESTUDIANTES AUTORREGULADOS}

Para Torrano y González (2004), la característica distintiva que algunas investigaciones encuentran en los estudiantes autorregulados es su participación activa desde el punto de vista metacognitivo, motivacional y comportamental.

Hay una coincidencia entre las características que se les atribuye a las personas autorreguladas con las atribuidas a los alumnos de alto rendimiento y de alta capacidad frente a los de bajo rendimiento (o con dificultades en el aprendizaje) que presentan déficit en esas variables (Reyero y Tourón, 2003).

Sin embargo, con un adecuado entrenamiento en esas dimensiones, todos los estudiantes pueden mejorar su grado de control sobre el aprendizaje y el rendimiento, y se pueden paliar muchas de las dificultades de aprendizaje que presentan, particularmente, los sujetos de rendimiento bajo.

He aquí una relación general de las características de los estudiantes autorregulados:

1. Conocen y saben emplear una serie de estrategias cognitivas (de repetición, elaboración y organización), que les van ayudar a atender a, transformar, organizar, elaborar y recuperar la información.

2. Saben cómo planificar, controlar y dirigir sus procesos mentales hacia el logro de sus metas personales (metacognición). 
3. Presentan un conjunto de creencias motivacionales y emociones adaptativas, tales como un alto sentido de autoeficacia académica, la adopción de metas de aprendizaje, el desarrollo de emociones positivas ante las tareas (p. ej., gozo, satisfacción, entusiasmo), así como la capacidad para controlarlas y modificarlas, ajustándolas a los requerimientos de la tarea y de la situación de aprendizaje concreta.

4. Planifican y controlan el tiempo y el esfuerzo que van a emplear en las tareas y saben crear y estructurar ambientes favorables de aprendizaje, tales como buscar un lugar adecuado para estudiar y la búsqueda de ayuda académica (help-seeking) de los profesores y compañeros cuando tienen dificultades.

5. En la medida en la que el contexto lo permita, muestran mayores intentos por participar en el control y regulación de las tareas académicas, el clima y la estructura de la clase (por ejemplo, cómo será evaluado uno mismo, los requerimientos de las tareas, el diseño de los trabajos de clase, la organización de los grupos de trabajo).

6. Son capaces de poner en marcha una serie de estrategias volitivas, orientadas a evitar las distracciones externas e internas, para mantener su concentración, su esfuerzo y su motivación durante la realización de las tareas académicas.

En resumen, si hay algo que caracteriza a estos alumnos es que se sienten agentes de su conducta, creen que el aprendizaje es un proceso proactivo, están auto motivados y usan las estrategias que les permiten lograr los resultados académicos deseados.

\section{APROXIMACIÓN DE LAS ESTRATEGIAS METODOLÓGICAS QUE FAVORECERÁN AL ESTUDIANTE AUTÓNOMO Y AUTORREGULADO}

\subsection{ESTRATEGIAS METODOLÓGICAS}

Hay muchas estrategias metodológicas a través de las cuales se puede favorecer e impulsar el aprendizaje autónomo y autorregulado; pero, dado que las estrategias metodológicas son como el punto de fusión entre los objetivos y los contenidos, y estos varían según el contexto y necesidades, no existe un método mejor que otro en términos absolutos. La "bondad" de los métodos depende de la situación concreta a la que se deseen aplicar: nivel educativo, área curricular, 
situación de aprendizaje... En términos relativos, una estrategia metodológica es más adecuada cuanto más se ajusta a las necesidades y maneras de aprender del alumno.

En el campo de las tecnologías de la información y la comunicación aplicadas a la educación, resulta interesante generar y validar estrategias de aprendizaje autorregulado que respondan a las necesidades diferenciales de los estudiantes que viven y trabajarán en un mundo altamente sofisticado en tecnologías de la información y comunicación (López, 2008).

Las estrategias metodológicas que se proponen en este trabajo para favorecer al estudiante autónomo y autorregulado como gestor de su propio aprendizaje consisten en el uso de diferentes recursos TIC. Siendo que las TIC se han convertido en los últimos años en toda una nueva metodología para ser aplicada en diferentes ambientes de aprendizaje.

Las tecnologías de información y comunicación han abierto nuevas posibilidades para la enseñanza y el aprendizaje. Su gran potencial se evidencia en la posibilidad de interacción, de comunicación, de acceso a información; es decir, se convierten en un medio interactivo y activo.

Estas tecnologías integradas a un entorno o ambiente de aprendizaje con diferente grado de virtualización, pone a disposición del docente canales de información y comunicación para promover formas distintas de enseñanza que desarrollen el aprendizaje autónomo del estudiante, lo cual plantea al docente el reto de aprovechar las TIC para hacer más consciente a los participantes de su proceso de aprendizaje y su papel en la regulación del mismo.

En este entorno de aprendizaje se encuentra el profesor como diseñador, dinamizador y orientador del proceso de aprendizaje y también el estudiante como sujeto que se compromete activamente y se siente responsable de su propio proceso de aprendizaje.

Así, el estudiante puede cursar asignaturas desde la Red Internet, enviar preguntas concretas o participar en grupos de discusión, navegar a través de las páginas electrónicas del curso y disponer de bibliografía, material didáctico, simulaciones y videos. El estudiante del siglo XXI, aquel que de una u otra manera interactúa todos los días con celular, domina el Ipod, accede a la Web, necesariamente tiene que haber desarrollado mayores y diversas habilidades, que quienes se educaron con lápiz y papel.

Todo esto le proporciona mayor riqueza de conocimientos, reduce la distancia geográfica con sus profesores y compañeros, e incrementa el tiempo de orientación que los docentes pueden dedicarle de manera personalizada. 


\subsection{RECURSOS TIC COMO ESTRATEGIA METODOLÓGICA}

\subsubsection{La pizarra digital}

La pizarra digital es un sistema tecnológico compuesto por un ordenador multimedia, un proyector y una pantalla sensible, con el que se pueden combinar los usos de las pizarras tradicionales con los recursos que nos proporcionan las TIC (herramientas de productividad y fuente inagotable de información multimedia e interactiva).

Ya hace algún tiempo que existe un importante interés por el uso de la pizarra como recurso en el aula, pero entonces este sistema era aún muy caro para poder generalizarse. Hoy existen nuevos dispositivos mucho más asequibles (proyectores y dispositivos sensibles), como el dispositivo eBeam, que con un tamaño muy reducido permite convertir cualquier superficie en una pizarra interactiva.

\subsubsection{Los WebQuest}

El modelo WebQuest fue creado por Bernie Dodge en 1995 y cuenta con miles de páginas en Internet, con propuestas de educadores de muchos países del mundo. Se interesa fundamentalmente en el diseño, implementación y evaluación de ambientes de aprendizaje basados en la Red, y en este campo tiene una amplia experiencia docente. El definió la WebQuest como "una actividad orientada a la investigación donde toda o casi toda la información que se utiliza procede de recursos de la Web" (Dodge, 1995).

Una WebQuest se construye alrededor de una tarea bien definida. Se trata de utilizar la información de recursos existentes en Internet y no de buscar información. La tarea puede implicar creación, crítica, resolución de problemas, enunciación de juicios, análisis o síntesis.

La WebQuest, por tanto, permite organizar y orientar el trabajo de estudiantes y profesores con relación al uso de tecnologías de la información.

Una WebQuest consiste en investigación guiada, con recursos principalmente procedentes de Internet, que obliga a la utilización de habilidades cognitivas elevadas, prevé el trabajo cooperativo y la autonomía de los alumnos e incluye una evaluación auténtica. Cada vez son más utilizadas como recurso didáctico por los profesores, puesto que permiten el abordaje de habilidades de manejo de información respondiendo así frente al desafío de educar en una sociedad altamente informatizada. 
Una WebQuest se construye alrededor de una tarea atractiva que provoca procesos de pensamiento superior. Se trata de hacer algo con la información. El pensamiento puede ser creativo o crítico, e implicar la resolución de problemas, enunciación de juicios, análisis o síntesis. La tarea debe consistir en algo más que en contestar a simples preguntas o reproducir lo que hay en la pantalla.

Una WebQuest tiene la siguiente estructura: introducción, tarea, proceso, recursos, evaluación, conclusión y autores.

Para desarrollar una WebQuest es necesario crear un sitio web que puede ser construido con un editor HTML, un servicio de blog o incluso con un procesador de textos que pueda guardar archivos como una página web.

Tipos de WebQuest

WebQuest de corta duración

Objetivo: La meta educacional de un WebQuest a corto plazo es la adquisición e integración del conocimiento de un determinado contenido de una o varias materias.

Duración: se diseña para ser terminado de uno a tres períodos de clase.

\section{WebQuest de larga duración}

Objetivo: extensión y procesamiento del conocimiento (deducción, inducción, clasificación, abstracción, etc)

Duración: entre una semana y un mes de clase

El uso de WebQuest aporta numerosas ventajas para el ejercicio y desarrollo de las habilidades de autorregulación de los alumnos:

- Permite que este elabore su propio conocimiento a la vez que realiza la tarea.

- Utiliza Internet con un objetivo claro y educativo.

- Emplea el tiempo de forma eficaz centrado en usar la información y no en buscarla.

- Permite el desarrollo de capacidades de análisis, síntesis y resolución de problema.

\subsubsection{Las actividades interactivas y tutoriales electrónicas}

Otra posibilidad es sentar a los alumnos a realizar una actividad en la que deben interactuar con la página web. Aquí cabe desde un tutorial que integre un tema completo con contenidos, actividades y evaluación, hasta una pequeña actividad que nos sirve para motivar al alumno o como práctica de autoevaluación. 
Además, el desarrollo de la autonomía y autorregulación no solo va a depender de la interacción del estudiante con el contenido a través del uso de las TICs en un ambiente de aprendizaje, sino también de las acciones tutoriales que motivan y ayudan al estudiante en la adquisición de creciente autonomía en el aprendizaje; además de la interacción con el tutor, está las interacciones entre participantes, los cuales pueden ejercer una influencia educativa sobre sus compañeros, asumiendo el rol de mediadores más expertos, promoviendo el intercambio o confrontación entre puntos de vista que, como vimos es importante en la autonomía intelectual, así como ejerciendo una regulación recíproca entre los participantes (Del Mastro, 2003).

\subsubsection{Trabajo colaborativo}

Web 2.0 es un término cada vez más popular y que identifica una especie de segunda generación de la web; una evolución de las aplicaciones tradicionales empleadas en la red que permite a los internautas participar de forma más activa e inmediata, suministrando, seleccionando y consumiendo los contenidos por ellos elegidos.

La web 2.0 se aleja del concepto estático que tenía la red, donde el usuario era fundamentalmente receptor de información, y se convierte en plataforma de servicios que fomentan la colaboración y el intercambio de información entre los usuarios.

La tecnología que emplea la Web 2.0 es variada, compleja y está en constante evolución: HTTP, JavaScript, XML, RSS, CSS... y especialmente AJAX. Pero los usuarios no tienen por qué ser expertos en estas tecnologías, ya que gracias a los editores y gestores de contenido el mantenimiento es tan fácil como escribir con un procesador de textos.

Han surgido numerosas herramientas del tipo Weblog, Wikis, redes sociales (networking), feeds, widgets, sistemas para insertar publicidad (AdSense, AdWords).

Los weblogs educativos o edublogs se han convertido en un recurso interesante para el aula que pueden aportar numerosas ventajas al proceso educativo. Por ejemplo la Wikipedia, los wikilibros, que están creando nuevos recursos educativos, originados por esta "inteligencia colectiva" permite abrir nuevas fuentes de información donde apoyarse los alumnos.

El uso educativo de los blogs está ya bastante extendido y existe un gran número de bitácoras dedicadas a la enseñanza, hasta el punto de crearse un término específico para designarlas, edublog. Los objetivos y temáticas son muy 
variadas, pero en esencia encontramos en la red tres tipos de blogs educativos, o mejor dicho, tres posibilidades de usar el blog:

- Los blogs de docencia para profesores, que permiten compartir y debatir experiencias de profesores de distintos centros y áreas geográficas.

- Los blogs de clase o de profesores para alumnos, que permite dirigir el proceso de aprendizaje de los alumnos, incluyendo actividades, enlaces de interés, aclaraciones y orientaciones para el estudio.

- Y los blogs de grupos de alumnos, que sirven para dar cohesión a una red de aprendizaje y realizar trabajos colaborativos.

Numerosas iniciativas han creado ya un gran número de blogs con contenidos y objetivos educativos. Además numerosas experiencias de su uso en el aula han creado espacios para facilitar el desarrollo de blogs educativos como: aulablog y Kalipedia y se han realizado numerosos estudios sobre las posibilidades de los blogs en la educación.

\section{CONCLUSIONES}

$1^{\mathrm{a}}$. Las estrategias de aprendizaje autorreguladas son acciones y procesos dirigidos hacia la adquisición de información o habilidades que involucran gestión, propósito y percepciones instrumentalizadas por los estudiantes. Los estudiantes autónomos y autorreguladores se caracterizan por tener diversas capacidades cognitivas y metacognitivas.

$2^{\mathrm{a}}$. La capacidad de autorregulación no es un esfuerzo aislado por parte del estudiante, sino que implica ayuda social y el uso activo de diferentes recursos (dimensión conductual), que le posibiliten el logro académico esperado y ser participe activo de su propio proceso de aprendizaje.

$3^{\mathrm{a}}$. Los recursos TIC ofrecen una gran variedad de ventajas para el aprendizaje autorregulado, en tanto estos medios particularmente exigen una gran iniciativa de parte del usuario. En el presente trabajo solo se ha considerado cuatro de ellos: La pizarra digital, los WebQuest, las actividades interactivas y tutoriales electrónicas y trabajo colaborativo: La Web 2.0.

\section{BIBLIOGRAFÍA}

BANDURA, A. (1982). Self-efficacy mechanism in human agency. American Psychologist, 37, 122-147.

BANDURA, A. (1987). Social foundations of thought and action: A social cognitive theory. Englewood Cliffs, NJ: Prentice Hall. (Trad. cast.: Pensamiento y acción. Fundamentos sociales. Barcelona: Martinez Roca). 
BROCKET RALPH Y HIEMTRA ROGER. (1993).El aprendizaje autodirigido en la educación de adultos. Barcelona: Paidós.

Del MASTRo, Cristina (2003). El aprendizaje estratégico en la educación a distancia. Lima: Fondo editorial PUCP. Serie: Cuadernos de Educación.

DodGE, B. J. (1995). WebQuests: A structure for active learning on the World Wide Web. The Distance Educator, 1(2).

Garza, Rosa María; Leventhal, Susana. (1998). Aprender como aprender. México. Editorial Trillas.

Hoyos VÁzQuEZ, GuilLERMO. (1998). Educación y autonomía en enfoques pedagógicos No. 14, Volumen 5 (1); enero -abril de 1998; Santafé de Bogotá D.C.

INSUASTY, LUIS DELFín. Aprendizaje autónomo en documento de apoyo técnico. UNAD-CAFAM. Santafé de Bogotá D.C. 1997.

LÓPEZ VARGAS, OMAR (2008). Desarrollo de la autorregulación en el aprendizaje con ambientes computacionales. Ponencia tic - cognición - aprendizaje.

Malagón, FÉlix Antonio (2005). Estrategias de aprendizaje para el tercer milenio. Escuela de Administración de Negocios EAN, Bogotá D.C.

Moos, D. C., \& AzEvEdo, R. (2007). Monitoring, planning, and selfefficacy during ..., Computers in Human Behavior doi:10.1016/j.chb.2007.07.001.

MoReno RAFAel y RAFAel J. (2007). Aproximación conductual a la noción de aprendizaje autónomo. Red estatal de docencia universitaria (REDU). En el Seminario Internacional 2-07: El desarrollo de la autonomía en el aprendizaje.

PARIS, S. G., \& Newman, R. S. (1990). Developmental aspects of self-regulated learning. Educational Psychologist, 8, 293-316.

PIAGET, J. (1975). Psicología de la inteligencia. Buenos Aires: Psique (Trabajo original publicado en 1947).

PINTRICH, P.R. (1986). Motivation and Learning Strategies Interactions with Achievement. Paper Presented at the College Classroom. Michigan: National Center for Research to Improve Postsecondary Teaching and Learning, the University of Michigan.

PINTRICH, P.R. (1995). Understanding Self-Regulated Learning. En P.R. Pintrich (Ed), New Directions for teaching and learning, No 63: Understanding Self-Regulated Learning. San Francisco: Jossey-Bass.

REYERO, M. Y TOURÓN, J. (2003). El desarrollo del talento: la aceleración como estrategia educativa. A Coruña: Netbiblo.

RiBeS, E. y LóPEZ, F. (1985): Teoría de la conducta. Análisis de campo y paramétrico. México: Trillas Tiscar, Lara: Blogs para educar. Usos de los blogs en una pedagogía constructivista. Revista Telos. No 65. Octubre / diciembre 2005.

Torrano Montalvo Fermín y María CARmen GonZÁlez Torres (2004). El aprendizaje autorregulado: presente y futuro de la investigación. Revista Electrónica de Investigación Psicoeducativa, 2 (1), 1-34. ISSN: 1696-2095 (2004).

VyGotsky, L. S. (1979). El desarrollo de los procesos psicológicos superiores. Madrid: Crítica (Trabajo original publicado en 1930).

ZIMMERMAN, B. J. (1986). A social cognitive view of self-regulated academic learning. Journal of Educational Psychology, 81(3), 329-339.

ZiMMERMAN, B. J. (1994). Dimensions of academic self-regulation: a conceptual framework for education. En D. H. Schunk y B. J. Zimmerman (Eds.), Self-regulation of learning and performance. Issues and educational applications. Hillsdale, NJ: Erlbaum. 